Gdańsk 2019, Nr. 41

https://doi.org/10.26881/sgg.2019.41.13

Andrzej Kątny

Universität Gdańsk

https://orcid.org/0000-00025611-8257

\title{
Quantifizierung der Nominalphrase durch Aspekt und Aktionsarten im Polnischen und Deutschen
}

In dem Beitrag werden einige Auffassungen zu den Aktionsarten im Polnischen, Deutschen sowie in der anglistischen Fachliteratur skizzenhaft dargestellt. Den Hauptteil des Artikels stellen Erwägungen zum Einfluss des grammatischen und lexikalischen Aspekts auf die Quantifizierung der Nominalphrase in beiden Sprachen dar. Die diskutierte Problematik wird mit einer Reihe von Belegen veranschaulicht.

Schlüsselwörter: Aktionsart, telisch, atelisch, Partitiv, Massennomina, nominale Quantifizierung

Quantification of nominal phrase in Polish and German through aspect and manners of action. - In the article different frames of manner of actions in the Polish and German languages as well as in English literature were presented. The main part of the article is devoted to consideration to influence of aspectuality (lexical and grammatical aspect) on nominal phrase quantification. This consideration is supported by the examples taken from the following languages: German, Polish and English.

Keywords: manner of action (Aktionsart), telic, atelic, partitive, mass nouns, nominal quantification

\section{Einleitung ${ }^{1}$}

In der Germanistik und Slawistik war die Grenzziehung zwischen Aspekt und Aktionsart unscharf. AGRELL (1908: 78) hat eine klare terminologische Grenzziehung vorgeschlagen:

„Unter Aktionsart verstehe ich [...] nicht die beiden Hauptkategorien des slawischen Zeitwortes, die unvollendete und die vollendete Handlungsform (das Imperfektivum und das Perfektivum) - diese nenne ich Aspekte. Mit dem Ausdruck Aktionsart bezeichne ich [...] Bedeutungsfunktionen der Verbalkomposita (sowie einiger Simplizia und Suffixbildungen), die genauer ausdrücken wie die Handlung vollbracht wird, die Art und Weise ihrer Ausführung markieren“.

Der Forscher weist auch darauf hin, dass die Bedeutung der Aktionsarten (im Weiteren: AA) aus der lokalen Funktion der Präfixe entstanden ist. Im Bereich der Slawistik hat die Untersuchung von IsAČENKo (1962: 385-418) breite Aufnahme gefunden. Neben Aspekt

1 Zum Teil stütze ich mich auf KĄTNY (2018). 
und AA unterscheidet er den Verbalcharakter als „ein Merkmal der inneren Verbbedeutung“ (ebd.: 398). Den Aktionsarten schreibt er drei notwendige Merkmale zu:

„1.Zusätzliche Modifizierung der Eigenbedeutung eines konkreten Verbs, [...]. 2. Formales Ausdrucksmittel: Anfügung eines modifizierenden Suffixes oder Präfixes an ein selbständig existierendes Verb (meist an ein Simplex). [...]. 3. Aspektmäßige Unpaarigkeit ${ }^{2}$ der Verben, welche Aktionsarten ausdrücken [...]. (ebd.: 386)

Im Deutschen gibt es eine weite und eine enge Auffassung von AA. Die enge Auffassung wird von STEINITZ (1981: 76) vertreten - AA „beziehen sich auf eine bestimmte Auswahl möglicher Modifikationen in den Bedeutungen von Vorgängen". AA sind eine Verbkategorie (und nicht eine Kategorie des Satzes) und „basieren auf der paradigmatischen Distinktion Simplex : morphologische Ableitung“; diese „Distinktion korreliert mit der semantischen aktionsartneutral : aktionsartspezifiziert“"(STEINITZ 1981: 76f.). Zu den Aktionsarten rechnet die Verfasserin nur ingressive und resultative Präfixableitungen (z.B. loslachen, auslernen, sich austoben), Suffixableitungen wie deuteln, plätschern und Funktionsverbgefüge vom Typ Präposition + substantivierter Infinitiv + Funktionsverb. Eine vergleichbare Auffassung findet man in ZIFONUN et al. (1997: 1860-1869). Darin wird zwischen "den allgemeineren Verbalcharakteren und den nur auf Präfixverben bezogenen Aktionsarten" differenziert. Die AA sind ein Wortbildungsphänomen: Sie „werden durch Verbalpräfixe gegenüber den Basisverben eingebracht, peripher auch durch das Suffix -(e)l(lächeln, streicheln)“ (ebd.: 1861). Unter Verbalcharakteren werden vier Verbtypen: telische (+Grenzphasenbezug), transformative (+Zustandsveränderung) und nicht-transformative (kursive und intransformative) Verben subsumiert. Die Verfasserin weist auch auf die Kombinierbarkeit der Telizität mit bestimmten Typen von Nominalphrasen hin:

„[... telische Verbgruppen werden mit ,individualisierten und nummerisch abgegrenzten ${ }^{\text {NP }}$ kombiniert, nicht mit , nicht-individualisierten ' bzw. ,kumulativen'; nicht telische verhalten sich genau umgekehrt“ (ZifOnUn et al. 1997: 1867).

In der weiten Auffassung werden die AA durch die Bedeutung des Verbs selbst, durch Affixe, syntaktische Konstruktionen und lexikalische Mittel zum Ausdruck gebracht. Als oberstes Gliederungskriterium der AA gilt das Merkmal grenzbezogen (telisch) vs. nichtgrenzbezogen (atelisch). In vielen Grammatiken und älteren Studien wurde die Bezeichnung perfektiv (auch terminativ oder punktuell) und imperfektiv (auch durativ oder kursiv) verwendet, was zu Missverständnissen geführt hat, weil mit den Termini perfektiv vs. imperfektiv die binäre Kategorie des Aspekts in den slawischen Sprachen bezeichnet wurde und wird. In der DUDEN-Grammatik (2016) werden zuerst telische, atelische und neutrale Verben unterschieden; zugleich wird richtungsweisend festgestellt:

„Die Aktionsart eines Verbs ist in vielen Fällen nicht ein für alle Mal festgelegt, sondern eher als ein Potential aufzufassen, das je nach dem Zusammenhang, in dem das Verb erscheint, die eine oder andere Richtung nehmen kann“" (2016: 417, Hervorhebung: A.K.).

Einfluss auf deren Bestimmung üben weitere Satzglieder aus: z.B. essen, trinken sind atelisch, aber ein Kilo Kirschen essen, zwei Glas Wein trinken - telisch. ANDERsson (1972) differenziert

2 Neuere Untersuchungen zeigten jedoch, dass es hier Ausnahmen gibt; vgl. dazu Avilova (1976); KĄTNY (1994). 
zwischen lexematisch grenzbezogenen (telischen), nichtgrenzbezogenen (atelischen) und neutralen Verben. Bei neutralen Verben (z.B. schreiben, bauen) handelt es sich um Verben, die „transitiv wie intransitiv gebraucht werden können“; beim transitiven Gebrauch werden sie als telisch eingestuft, „wenn sie ein effiziertes Objekt nehmen“ (ANDERsson 1972: 41). Die Untersuchung erfolgt auf der lexematischen Ebene (Subjekt + Prädikat + eine Ergänzung), der rektionellen Ebene II (mit weiteren Ergänzungen, Angaben) und der prädikatsverbindenden Ebene III. Im Satz (3) ist das Verb sich freuen lexematisch atelisch, hier (im Satzgefüge - Ebene III) wird es als telisch gedeutet.

(1) den Wagen ziehen [lexematisch atelisch]

(2) den Wagen auf den Hof ziehen [Ebene II: telisch]

(3) Als er dies erfuhr, freute er sich. [Ebene III: telisch]

In der anglistischen Fachliteratur wird die Bezeichnung Aktionsart kaum verwendet; stattdessen spricht man von vier Verbklassen, Situationstypen oder Zeitschemata (time schemata), die aufVENDLER (1957) zurückgehen und über Merkmalskombination unterschieden werden (als Beispiele führe ich Belege aus dem Deutschen an):

(a) states (Zustände) [-dynamisch, -telisch, +durativ]: wissen, lieben, schlafen;

(b) activities (Aktivitäten, Prozesse) [+dynamisch, -telisch, +durativ]: Suppe essen, schreiben, Kreise zeichnen, laufen;

(c) accomplishments (ausgedehnter Zustandswechsel) [+dynamisch, + telisch, +durativ]: einen Kreis zeichnen, die Suppe essen, einen Aufsatz schreiben, ein Haus bauen, zur Post laufen;

(d) achievements (Ereignisse, punktueller Zustandswechsel) [+dynamisch, +telisch, -durativ]: etwas finden, aufwachen, sterben, entdecken, das Ziel erreichen, eine Grenze überqueren.

Die Klassifikation von VendLER bezieht sich nicht nur auf Verben, sondern auch auf Verbalphrasen (insbesondere bei accomplishments und z.T. bei achievements). Die Zuordnung zu den einzelnen Klassen ist kontextabhängig und hängt u.a. vom Typ des Objekts (Individualund Massennomina, Numeralia), Direktional-, Iterativ- und Daueradverbialien ab, worauf u.a. VERKUYL (1972), KRIFKa (1989) und FiLIP (1999) hingewiesen haben.

VERKUYL $(1972,1993)$ unterscheidet zwischen viewpoint oder grammatical aspect [Perspektivenaspekt] und „inner/telicity“ oder „predicational aspect“; der letztere Begriff basiert auf der Unterscheidung zwischen telischen (terminativen in seiner Terminologie) und atelischen (durativen) Prädikaten. Ausschlaggebend für die Zuordnung der Situationen zu den telischen oder atelischen ist das Zusammenspiel der aktionalen Bedeutung des Verbs und seiner Argumente (= kompositionelle Telizität). Der Forscher unterscheidet zwischen spezifischen $[+\mathrm{SQA}]^{3}$ und unspezifischen Argumenten/Objekten [-SQA]. Bei den spezifischen quantifizierten Objekten handelt es sich um zählbare Maßeinheiten. VERKUYL formuliert einige Regeln, die für Verben der Konsumption, Kreation sowie der Bewegung gelten (darunter eine vereinfachte und stark reduzierte Darstellung):

(4) ein atelisches Verb $+[+$ SQA $]$ ergibt eine telische VP

(5) ein atelisches Verb + [-SQA ] ergibt eine atelische VP

$3+\mathrm{SQA}=$,Specified Quantity of A“ - in der Terminologie von KRIFKA (vgl. 3.1) werden diese Nomina als gequantelt bezeichnet. -SQA = „Unspecified Quantity of A” - nach KRIFKA sind diese Nomina kumulativ. 
Einige z.T. modifizierte Beispiele aus VerkuYL (1993: 15f.) mögen die Regeln (4-5) veranschaulichen (vgl. 6-11).

(6) They ate cheese, sandwiches.

(7) They ate three sandwiches.

(8) They drank whisky.

(9) They drank a litre of whisky.

(10) He walked.

(11) He walked a kilometr / to the station.

Diese Regeln kann man auch auf das Deutsche sowie auf andere germanische Sprachen anwenden; dabei müsste jedoch der über die Satzgrenze hinausgehende breitere Kontext berücksichtigt werden. In den slawischen Sprachen ist der Aspekt grammatikalisiert und der Sprecher wählt automatisch die entsprechende Form des Verbs, sodass diesen Fragen weniger Aufmerksamkeit gewidmet wird. Für kontrastive und Übersetzungszwecke kann aber die kompositionelle Telizität brauchbar sein; einige vergleichbare Beispiele aus dem Deutschen (mit Übersetzungen ins Polnische) führe ich in (12-13a) an:

(12) Wein trinken (pićlPF wino)

(12) a. den Wein, ein Glas Wein trinken (wypićp wino, kieliszek wina)

(12) b. täglich ein Glas Wein trinken (wypijać ${ }^{\text {IPF }}$ codziennie kieliszek wina)

(13) Brot essen (jeśćc ${ }^{I P F}$ chleb)

(13) a. das Brot essen (zjeść ${ }^{\mathrm{PF}}$ chleb)

Massennomina sind nicht spezifisch (vgl. 6, 8, 12, 13), durch zählbare, bestimmte Maßeinheiten (z.B. den bestimmten Artikel, Demonstrativpronomina, quantifizierende Pronomina, Numeralien, Behälterbezeichnungen) werden sie zu spezifischen Argumenten (vgl. 7, 9, 12a, 13a). Im Satz 13 wird chleb als MN verwendet, dagegen wird es in 13a. dank der telisierenden Wirkung des perfektiven Aspekts zum gequantelten Substantiv (d.h. zum IN); mit der gleichen Verschiebung haben wir es in 12 und 12a. zu tun (bei wino).

Alle polnischen (slawischen) Verben sind aspektual markiert; Aspektpaare bildet nur ein Teil der Verben (in bestimmten Bedeutungsvarianten). Im Zusammenhang damit gibt es Perfektiva und Imperfektiva tantum. Außerdem gibt es im Polnischen (und in der Slavia) biaspektuale Verben, z.B.: anulować ,annulieren', amputować ,amputieren', kanonizować ,kanonisieren', ratyfikować ,ratifizieren' u.v.a. $\mathrm{Zu}$ anderen Unregelmäßigkeiten, die vielen Ausländern und Forschern Probleme bereiten, gehören z.B. allgemeinfaktische Imperfektiva (Imperfektiva, die als Perfektiva verwendet werden), das historische Präsens und zum Teil die (k)overte Ingressivität. Die beiden letzteren Probleme werden im Folgenden andiskutiert und bilden zugleich einen Übergang zum Hauptthema meines Artikels.

\section{2. (K)overte Ingressivität}

Im Polnischen und den meisten slawischen Sprachen wird die Ingressivität im Prinzip overt durch bestimmte Präfixe ${ }^{4}$ (z.B. im Polnischen durch $\left.z a-, p o-\right)$, Umschreibung mit Phasenverben

4 Mit diesen Präfixen wird das Verb perfektiviert (= perf. Aspekt); in vielen Fällen wird die Verwendung eines perfektiven Verbs im Polnischen durch den Kontext erzwungen (vgl. die Beispiele a und a'). 
wie zaczać, beginnen' oder mit Funktionsverbgefüge (FVG) ausgedrückt. Im Deutschen dagegen wird die Ingressivität viel seltener mit Präfixverben oder FVG zum Ausdruck gebracht; der Anfang der Handlung kann dem Kontext entnommen werden:

(14) a. Lecz w tej chwili zaszeleściły ${ }^{\mathrm{PF}}$ liście pobliskich mirtów ... (Quo, 320)

a $\quad$ Lecz w tej chwili * szeleściły ${ }^{\mathrm{IPF}}$ liście pobliskich mirtów ...

b. In diesem Augenblick rauschten die Blätter der nächsten Myrte. (Boliński, 376)

c. Da raschelte es im Myrtenlaub. (Ettlinger, 288)

(15) a. Nagle zatrąbil ${ }^{\mathrm{PF}}$ dwukrotnie klakson. (Asche, 8)

a' Nagle *trąbif ${ }^{\text {IPF }}$ dwukrotnie klakson.

b. Plötzlich hupfte es zweimal. (S. 7)

(16) a. Wstał ${ }^{\mathrm{PF}}$, zapukat ${ }^{\mathrm{PF}}$ do sąsiednich drzwi. (Iwasz., 16)

a' Wstal ${ }^{\mathrm{PF}},{ }^{*}$ pukał ${ }^{\mathrm{IPF}}$ do sąsiednich drzwi

b. Er erhob sich, klopfte an die Tür des benachbarten Zimmers. (S. 17)

(17) a. Ich setzte mich ins Zimmer und rauchte. (fremd, 201)

a' Usiadłem ${ }^{\mathrm{PF}}$ w pokoju i ${ }^{*}$ paliłem ${ }^{\mathrm{IPF}}$ papierosa.

b. Usiadłem ${ }^{\mathrm{PF}} \mathrm{w}$ pokoju i zapaliłem ${ }^{\mathrm{PF}}$ papierosa. (S. 130) a b e r:

(18) a. Ich saß ... und rauchte.

b. Siedziałem ${ }^{\mathrm{IPF}}$ i paliłem ${ }^{\mathrm{IPF}}$ papierosa. (Übers.: A.K.)

Wie die Beispiele zeigen, kann im Deutschen der Beginn der Handlung durch den engeren oder weiteren Kontext bestimmt sein; eine wichtige Rolle erfüllt hierfür - neben den Adverbialphrasen, die den Anfang bzw. eine neue Handlung bezeichnen (z.B. plötzlich, in diesem Augenblick, da) - die natürliche Sequenz der Handlungen (oft markiert durch ein telisches Verb). Im Polnischen gibt es nur vereinzelt Fälle, in denen Ingressivität nicht explizit versprachlicht wird, sondern sich aus dem Kontext ergibt. In der von DICKEY angebotenen Übersetzung wird das imperfektive szlochat, (er) schluchzte' durch das Phasenverb + ing-Form wiedergegeben. In meiner Übersetzung (19c) verwende ich die durative Form des Verbs (= lexematisch atelisch), da der Kontext die ingressive Nuance beisteuert und das Verb als aufschluchzen oder beginnen zu schluchzen gedeutet werden kann.

(19) a. Jakub usiadlPF na łóżku i szlochał ${ }^{\mathrm{IPF}}$.

b. 'Jakub sat down on the bed and started sobbing' (DICKEY 2000: 211, zit. nach IVANČEV 1961: 42; Nałkowska, Niecierpliwi)

c. Jakub setzte sich auf das Bett und schluchzte. (Übers.: A.K.)

Berger (2013: 60f.) hat im Parallelkorpus Parasol einige weitere Beispiele für die „Verwendung des imperfektiven Aspekts in der Abfolge von Ereignis und Verlauf" gefunden; als Ausgangstext diente ihm der Roman von Kundera und seine Übersetzungen ins Russische und ins Polnische - im Folgenden führe ich nur einige Beispiele für das Polnische ${ }^{5}$ (S. 61) an und übersetze sie ins Deutsche:

(20) a. Dubczek powrócil ${ }^{\mathrm{PF}}$ do Pragi i czytall ${ }^{\mathrm{IPF}}$ przez radio swe przemówienie.

b. Dubček kehrte nach Prag zurück und hielt im Rundfunk seine Ansprache. (Übers.: A.K.)

(21) a. ... chłopak wywichną ${ }^{\mathrm{PF}}$ sobie podczas pracy rękę w ramieniu i wy ${ }^{\mathrm{IPF}} \mathrm{z}$ bólu.

5 Im Ausgangstext steht jeweils in der Handlungssequenz nach dem perfektiven ein imperfektives Verb; es ist nicht auszuschließen, dass der Übersetzer sich streng an diese Form hielt. 
b. ... der Junge hatte sich während der Arbeit den Arm verrenkt und heulte vor Schmerzen.

(Übers.: A.K.)

Im Tschechischen, einer westslawischen Sprache mit ausgebautem Aspektsystem und morphologisch markierten AA, erscheint in der Handlungssequenz nach einem Verb im perfektiven Aspekt ein imperfektives Verb. Darauf hat zuerst IvANČEv (1961) aufmerksam gemacht und diese Erscheinung als Contextually-Conditioned Imperfective Past ${ }^{6}$ (DiCkey 2000: 203) bezeichnet:

(22) Sedl ${ }^{\mathrm{PF}}$ si a psal $^{\mathrm{IPF}}$

'He set down and started writing'.

(DiCKey 2000: 204, nach IVANČEv 1961: 6)

\section{Das historische Präsens}

Das historische Präsens wird in der Stilistik als Mittel zur Belebung und Abwechslung des Stils bezeichnet; nach der Textgrammatik von WEINRICH (1993: 217) habe das historische Präsens das Ziel, „einer Erzählung ein größeres Maß an Unmittelbarkeit, Lebhaftigkeit und Spannung zu verleihen." In der Tempusforschung werden hauptsächlich die Vergangenheitsfunktion und die Vergegenwärtigung unterstrichen. In Duden (2016: 517) werden der Vergangenheitsbezug und der „nach dem Diskurstyp und dem weiteren Kontext“ variierende stilistische Effekt erwähnt; in erzählenden Texten sei diese Form so stark konventionalisiert, „dass der Vergegenwärtigungseffekt abgeschwächt erscheint“ (ebd.).

Viel seltener wird auf die aspektuelle oder aktionsartliche Funktion des historischen Präsens hingewiesen - darauf machten u.a. Koschmieder (1934/1987), Kotin (2015) und LeIss (2000) aufmerksam. Nach LeIss (2000: 73) weist das historische Präsens zwei relevante Markmale: [+VERGANGENHEITSBEZUG] und [+PERFEKTIVITÄT] auf. Ebenfalls Koтin (2015: 11) schreibt diesen Formen einen „Perfektivierungseffekt“ zu: In den slawischen Sprachen werden „die präsentischen Verben des imperf. Aspekts obligatorisch perfektiv gelesen“ (S. 10), im Deutschen, wo der Aspekt kovert kodiert ist, wird dieser Effekt „durch die jeweilige Aktionsart-Lesart manifestiert" (ebd.). Ich stimme Kotin (2015) zu, dass diese perfektive Kodierung nicht allen Verben im historischen Präsens zugeschrieben werden kann.

(23) a. Siedzę $e^{\mathrm{IPF}}$ wczoraj przy biurku [... a wtem nagle Antek wpada ${ }^{\mathrm{IPF}}$ do pokoju.

b. Ich sitze gestern am Schreibtisch [...], da stürzt plötzlich Anton ins Zimmer.

(24) a. Gestern gehe ich in die Stadtbücherei. Da steht plötzlich der Nikolaus vor mir (Ek 1992: 27)

b. Idę $e^{I P F}$ (sobie) wczoraj do biblioteki miejskiej, a tu nagle staje ${ }^{\text {IPF }}$ przede mną święty Mikołaj. (Übers.: A.K.)

In $(23,24)$ haben wir es mit dem Situationstyp „Währen“ (Hintergrund) - „Eintritt“ (vgl. Koschmieder [1934] / 1987: 83) zu tun; der letztere wird normaleweise in der Slavia durch den perfektiven Aspekt, im historischen Präsens dagegen durch den imperfektiven Aspekt ausgedrückt.

6 Im tschechischen Terminus von Ivančev kommt noch die Bezeichnung ingressiv vor; DiCKey (2000: 203) lässt sie in seiner Übersetzung bewusst weg, weil er diesem Phänomen auch andere Funktionen zuschreiben will. 


\section{Aspekt und Quantifizierung}

\subsection{Nominale Referenz}

Einleitend sind einige Überlegungen zu den Massennomina (MN; mass nouns, auch Kontinuativa genannt) und den Individualnomina (IN; count nouns) unentbehrlich. Bei den IN handelt es sich um diskrete, zählbare Objekte. Unter MN verstehe ich, in Anlehnung an JESPERSEN ([1924] /1965: 198), „words which do not call up the idea of some definite thing with a certain shape or precise limits".

In der Klasse von MN kann man neben Abstrakta (auf die ich nicht weiter eingehen möchte) noch zwei weitere Subklassen unterscheiden: Stoffnomina (Gold, Eisen, Wolle, Bier, Wein) und Kollektivnomina (Vieh, Obst, Schmuck, Müll). Während IN mit Numeralia unmittelbar verbunden sein können (z.B. zwei Bleistifte, drei Hefte), sind die MN mit den Numeralia direkt nicht verbindbar ${ }^{7}{ }^{*}$ drei Gold, ${ }^{*}$ zwei Honig); sie können sich nur mit einem quantifizierten Nomen (quantN) verbinden, wobei dem quantN ein Numerale vorausgehen muss: drei Unzen Gold, zwei Gläser Honig. Unter den quantN lassen sich mehrere Gruppen unterscheiden (vgl. LöBEL 1986: 12-15).

- Messkonstruktionen (Mensurativa)

a) Standardmaße: z.B. Elle (Stoff), Hektar, Kilo, Pfund, Klafter (Holz), Maß (Bier), Unze (Gold)

b) Unbestimmte Mengenangaben: z.B. Dosis (Medizin), Prise (Salz), Schluck (Tee), Schuss (Weinbrand)

- Zählkonstruktionen (das nicht Zählbare wird zählbar gemacht)

a) Behälternomina: Fass, Glas, Flasche, Kiste, Korb, Sack,

b) Numerativa: Blatt, Bogen (Papier), Brocken, Bündel, Haufen, Knäuel (Wolle), Laib, Riegel, Tafel (Schokolade), Scheibe (Wurst), Schnitte, Zehe (Knoblauch).

Den Unterschied zwischen den IN und MN veranschaulicht die in Anlehnung an KRIFKA (1989a: 4) erstellte Tabelle (die Wortbelege stammen von mir) (Tabelle 1).

Die MN und IN unterscheiden sich voneinander durch das Merkmal der Zählbarkeit; sie weisen aber auch ein gemeinsames Merkmal auf: das der Kumulativität, d.h. die IN im Plural und MN sind kumulativ; der Begriff der Kumulativität geht auf QuiNE (1960: 91) zurück: „any sum of parts which are water ist water“.

KRIFKA (1989, 1989a) knüpft u.a. an VERKUYL (1972) an und sieht einen Zusammenhang zwischen der „Nominalreferenz von Verbargumenten“ und der „Zeitkonstitution von

\footnotetext{
Es gibt hier einige Ausnahmen bei Behälternomina, z.B. unter zwei Bier werden zwei Flaschen oder zwei Glas Bier und unter zwei Kaffee, Tee - zwei Tassen Kaffee, Tee verstanden; die Menge oder das Quantum ergibt sich aus der Situation und/oder Kenntnis der Gäste, Konsumenten und ist stark beschränkt. ${ }^{*}$ Zwei Wein im Sinne von zwei Glas Wein sind nicht korrekt. Zwei Mehl (vgl. Tabelle 1) klingen ungewöhnlich; vorstellbar ist aber die Situation, wo der Großabnehmer zwei Sack Mehl kaufen will und seinen Wunsch in gekürzter Form äußert. Wichtig in diesem Kontext ist die Unterscheidung zwischen Arten- und Maß-Klassifikator, was LYONS (1983: 89f.) an zwei Beispielen veranschaulicht: die Phrase "three whiskies“ kann im Satz We only stock three whiskies als "Art/Typ" und im Satz I only drank three whiskies in the course of the evening als "Quantum/Portion“ verstanden werden.
} 
komplexen Verbausdrücken“; er unterscheidet dabei zwischen der kumulativen ${ }^{8}$ und gequantelten Referenz (KRIFKA 1989: 228ff.); zu der ersteren gehören z.B. Milch, Bier, Schnaps, Mehl, Reis, Äpfel, Kartoffeln, Apfelmus, Marmelade, Gold und zu der letzteren: die Milch, ein Glas, eine Flasche Wein, drei Pfund Marmelade, ein Kilo Äpfel, drei Barren Gold. Sie können also gezählt und gemessen werden.

Tabelle 1.

\begin{tabular}{|l|l|l|}
\hline \multicolumn{2}{|c|}{ Individualnomen } & \multicolumn{1}{c|}{ Massennomen } \\
\hline \multicolumn{1}{|c|}{ Singular } & \multicolumn{1}{c|}{ Plural } & \multicolumn{1}{c|}{ Transnumeral } \\
\hline ein Roman & zwei Romane & ?zwei Mehl \\
\hline${ }^{*}$ viel Roman & viele Romane & viel Mehl \\
\hline${ }^{*}$ etwas Roman & *etwas Romane & etwas Mehl \\
\hline $\begin{array}{l}\text { jeder Roman } \\
\text { *aller Roman } \\
\text { *mehr Roman }\end{array}$ & $\begin{array}{l}\text { *jede Romane } \\
\text { alle Romane } \\
\text { mehr Romane }\end{array}$ & $\begin{array}{l}\text { ?jedes Mehl } \\
\text { alles Mehl } \\
\text { mehr Mehl }\end{array}$ \\
\hline
\end{tabular}

Massennomina sind nicht-spezifisch (kumulativ, atelisch). Durch zählbare Maßeinheiten (den bestimmten Artikel, Numeralia, Behälterbezeichnungen) werden sie zu spezifisch quantifizierten (gequantelt, telisch). Hier findet eine Verschiebung (shift) statt, d.h. ein MN wird einem IN ähnlich. KRIFKa (1989a: 96) erkennt Parallelen zwischen den nominalen Distinktionen (MN : IN) und solchen im Verbalbereich und überträgt die Begriffe der kumulativen und gequantelten Referenz auf die Verben; atelische Verben sind kumulativ, telische - gequantelt.

An dieser Stelle muss ich noch stichwortartig den Ansatz von Elisabeth LeIss (1992: 48ff.) erwähnen; sie unterscheidet bei der Beschreibung der Verben (der Aktionsarten, der Aspektualität) zwischen der Außenperspektive (Grenzbezogenheit) und Innenperspektive (Nichtgrenzbezogenheit), was zu zwei Klassen von Verben führt: zu innen- und außenperspektivierenden. Die innenperspektivierenden (atelischen) Verben sind kumulativ und die außenperspektivierenden (telischen) sind gequantelt. Die Forscherin wendet sich in einer weiteren Monographie (LeIss 2000) der Quantifikation von Objekten und der Telisierung von Ereignissen im Althochdeutschen und Gotischen zu.

\subsection{Zur Genitiv/Akkusativ-Alternierung}

Die durch den Genitiv (in Opposition zum Akkusativ) ausgedrückte Bedeutung kann man als unbestimmte Quantität oder als quantitative Indeterminiertheit (GLADROw 1979: 162) bezeichnen; auf diese Funktion des Genitivs hat schon früher u.a. JAKOBSON aufmerksam gemacht:

\footnotetext{
8 Birnen und Birnen ergeben Birnen (= kumulativ); dagegen drei Kilo Birnen und drei Kilo Birnen ergeben nicht drei Kilo sondern sechs Kilo (= gequantelt).

9 Zugelassen (akzeptabel) bei dem sogen. Sortenplural: jede Sorte Mehl.
} 
„Der G(enitiv - A. K.) an sich besagt nur, daß der Umfang der Teilnahme des Gegenstandes am Sachverhalt der Aussage geringer als sein gesamter Umfang ist. In welchem Maße der Umfang des Gegenstandes beschränkt wird, das bestimmt der sprachliche oder der außersprachliche Kontext“" (JAKOBSON [1936] 1971: 38).

Der alternative Gebrauch von Genitiv und Akkusativ kommt z.B. bei Verben mit possessiver Bedeutung im weiteren Sinne vor, z.B. dać [geben], dostać [erhalten, kriegen], kupić [kaufen], sprzedać [verkaufen], ukrasśc [stehlen], przynieść [bringen], przywieźć [herbringen]; bei konsumptiven Verben jeść [essen], pić [trinken]) und einigen weiteren.

(23) a. (On) pil ${ }^{\text {IPF }}$ kawę-AKK i jadilipF chleb-AKK

b. Er trank Kaffee-AKK und aß Brot-AKK

In den Sätzen (23a,b) kommen kumulative Substantive (Massennomina), in (24a,b) - gequantelte Substantive (Individualnomina) vor. Die Quantelung der Substantive ${ }^{10}$ wird in $24 a$ durch den perfektiven Aspekt herbeigeführt (erzwungen). Im Deutschen wird sie hier durch den bestimmten Artikel bezeichnet.
a. (On) wypif ${ }^{\mathrm{PF}}$ kawę-AKK
i $\quad$ zjadP $^{\mathrm{PF}}$ chleb-AKK
b. Er trank den Kaffe-AKK
und $a ß \quad$ das Brot-AKK
c. 'Er hat den Kaffe (aus)getrunken und das Brot aufgegessen'

Beim partitiven Genitiv können nur Substantive im Singular im Falle der MN oder im Plural - bei zählbaren Nomina (IN) vorkommen (vgl. 25a). Im Singular (vgl. 26a) sind sie dagegen ausgeschlossen:

(25) a. (On) wypiP ${ }^{\mathrm{PF}}$ kawy-GEN.SG, zjadPPF chleba-GEN.SG i ogórków-GEN.PL

b. 'Er hat vom Kaffe getrunken, etwas Brot und Gurken gegessen'

(26) a. On kupiPF (sobie) * cytryny / cytryn.

b. Er kaufte sich ${ }^{*}$ Zitrone-GEN.SG Zitronen-GEN.PL

Der Partitive Genitiv kann nur bei den perfektiven Verben stehen, die Verbindung mit den imperfektiven Verben ist unzulässig; WierzbickA (1967: 2238) formuliert dies folgendermaßen: „When combined with certain imperfective verbs, the object does not allow the specification by a modifier of measure (quantity)“ - vgl. Beispiel 27 und 28, zit. nach WieRZBICKA:

(27) * On jadł w tym momencie trochę kaszy ( ${ }^{*} \mathrm{He}$ was then eating some porridge).

(28) * On pił w tym momencie szklankę wody (trochę wody) ( ${ }^{*}$ He was then drinking a glass of water (some water).

(29) a. ${ }^{*}$ On pillPF kawy i jadlIPF (trochę) chleba.

b. $\quad{ }^{*}$ Er trank Kaffee-GEN.SG und aß (etwas) Brot-GEN.SG

(30) a. Piotr kupil ${ }^{\mathrm{PF}} /{ }^{*}$ kupowal ${ }^{\text {IPF }}$ gruszek.

b. Peter kaufte / ${ }^{*}$ kaufte Birnen-GEN.PL

10 Im Bulgarischen, einer slawischen Sprache mit dem voll ausgebauten postponierten definiten Artikel, wird diese Quantelung obligatorisch durch den definiten Artikel bezeichnet (das Beispiel entnehme ich FiLIP 1999: 228):

Toj izpi kkafe / kafeto.

He.NOM PREF.drink.PAST * ${ }^{*}$ coffee.SG.ACC / coffee.DF.SG.ACC

'He drank up (all) the coffee.' 
Der Gebrauch des Genitivs ist nicht zwingend; wie schon oben angedeutet, steht der Akkusativ, wenn der Gegenstand als Ganzes der Handlung unterzogen wird, der Genitiv dagegen dann, wenn sich die Handlung auf einen Teil des Objekts bezieht. Die Hauptfunktion des Genitivs kann man als Partitivität/Indefinitheit bezeichnen.

Der Verbindung des Akkusativs mit dem Verb im perfektiven Aspekt bei inkrementellen Argumenten wird in vielen Studien die determinierende Funktion zugeschrieben - d.h. bei der Übersetzung ins Deutsche, Englische sollte das Substantiv mit dem bestimmten Artikel stehen (so u.a. Birkenmaier 1979, Abraham 1997, SADZiński 1995/96).

WierzBiCKA (1967: 2243) zeigt an zwei Beispielen mit Verben im perfektiven Aspekt unterschiedliche Determinierung des Akkusativs:

(31) Jan nakarmił dzieci $=$ John has fed THE children, however

(32) Oni zbudowali nową szkołę = They have built A new school.

und zieht die folgende Schlussfolgerung:

„In combination with verbs which signify the causation of existens ('to build', 'to write', 'to create' etc.) the translation with the indefinite article would be almost a rule. Yet, in this case the article signifies the number: one“(WIERZBICKA 1967: 2243f.)

Es gibt aber auch Forscher, die diese Meinung nicht voll unterstützen, so z.B. Wolfgang GLADROW (1979: 156), der nach der Analyse von einigen Übersetzungen „nicht in allen Fällen die determinierte Artikelform“ vorgefunden hat. „,[Der Akkusativ] bezeichnet zwar häufig eine bestimmte Quantität, lässt dieses Merkmal aber prinzipiell unausgedrückt“ (ebd.: 156f.).

Fleischhauer und Czardybon (2016: 177) argumentieren, dass beim perfektiven Aspekt die inkrementellen Argumente nicht unbedingt mit dem definiten Artikel stehen müssen:

(33) Ona z-jadła kanapk-ę.

She Z-eat.PAST sandwich-ACC 'She ate a/the whole sandwich.'

Dieses Problem muss also noch weiter untersucht werden, wobei unterschiedliche Kontexte mit inkrementellen Verben, unter Bezugnahme auf die Wortfolge und den Akzent, berücksichtigt werden müssten; Korpusbelege aus dem Deutschen und Englischem sowie Parallelübersetzungen könnten hier zu mehr Klarheit beitragen.

\subsection{Kumulative AA}

Die kumulative AA drückt eine in Einzelakte gegliederte Handlung aus. Bei transitiven Verben, die sich obligatorisch mit einem Objekt im partitiven Genitiv (Singular bei MN und Plural bei IN) verbinden, wird die im Ergebnis dieser Handlungen angesammelte große Menge hervorgehoben.

Diese Verben werden mit dem Präfix na-von imperfektiven ${ }^{11}$ einfachen Verben (34a) und von sekundären Imperfektiva (34b) abgeleitet (dabei kann es sich um intransitive und transitiven Verben handeln). Sie sind Perfektiva tantum.

11 Als eine Ausnahme ist hier nakupić zu verstehen, das vom perf. Verb (kupić) abgeleitet wird. 
(34) a. nadyktować listów-PL, nałowić ryb-PL, nakraść pieniędzy-SG viele Briefe diktieren, viele Fische fangen, eine Menge Geld stehlen

b. naprzywozić zboża-SG viel Getreide heranfahren

Der partitive Genitiv kann zusätzlich um eine Mengenangabe (Partitiv-Quantoren) vom Typ wiele, dużo 'viele', mnóstwo 'Menge' erweitert werden; eine Verbindung mit Totalisatoren (wszystkie, wszyscy 'alle, sämtliche', każdy 'jede') oder Numeralien ist ausgeschlossen, da Partitivquantoren mit Totalisatoren und Numeralien nicht kompatibel sind.

(35) a. nadyktować *wszystkie / *dziesięć listów

b. viele *alle / ${ }^{*}$ zehn Briefe diktieren

(36) a. Potem mebli nazwozil. (Maria, 36)

b. Dann hat er Möbel angekarrt. (43)

(37) a. Miron kaufte ihr Hüte, Strümpfe, Wäsche, Schmuck. (Orph., 241)

b. Miron nakupił jej kapeluszy, pończoszek, bielizny i biżuterii. (290)

(38) a. Maciejunio własnoręcznie naznosił słoików z miodem, konfiturami, sokami. (Przedw.)

b. Maciejunio brachte eigenhändig Gläser mit Honig, Konfitüren, ... (139)

Wie den Beispielen zu entnehmen ist, übernimmt der Nullartikel im Deutschen die Funktion, die im Polnischen durch die na-Verben samt ihren obligatorischen Ergänzungen signalisiert wird. Der Nullartikel dient in diesen Sätzen zur Bezeichnung von Nicht-Gesamtheit und ist "mit allen Formen, die Nichtgesamtheit bezeichnen, austauschbar" (VATER 1979: 110).

\section{Zusammenfassung}

Mit dem Beitrag wollte ich auf unterschiedliche Forschungstraditionen im Bereich der Aspektualität und auf eine differenzierte sowie stellenweise verwirrende Terminologie hinweisen. Diese Unterschiede und andere Herangehensweisen sind primär durch die Sprachsysteme bedingt: Der Aspekt stellt in den slawischen Sprachen eine obligatorische morphologische Kategorie dar; in den germanischen Sprachen werden die durch den Aspekt und die Aktionsarten ausgedrückten Inhalte auf der Satz- und Textebene (in der Regel kovert) kodiert. Während in den slawischen Sprachen der Aspekt und sein Zusammenspiel mit den anderen Verbkategorien im Vordergrund stehen, erweitert die anglistische Forschung ihren Blick auf Nomina und Adverbialien sowie deren Einfluss auf die aktionale Deutung des Verbs auf der Satzebene.

Die von Leiss (2000: 149, 152, 185-187) für das Gotische und das Althochdeutsche festgestellte Genitiv-/Akkusativ-Alternierung bei perfektiven Verben lässt sich im Polnischen und im Allgemeinen auch in der Slavia (mit einigen Abweichungen, z.B. im Tschechischen - dazu u.a. FILIP 1999) beobachten:

„Der partitive Genitiv kodiert nichtzählbare, indefinite Teile eines definiten Ganzen. Gerade in Kombination mit perfektiven Verben erhält ein Genitivobjekt diese partitive Qualität (vgl. Kap. 1). Es ist klar 
ersichtlich, daß die definite Bedeutungskomponente von Partitivität durch das perfektive Verb beigesteuert wird, während das Teilmerkmal der Indefinität vom Genitiv eingebracht wird“ (LEIss 2000: 186).

Meine Analyse zeigt und bestätigt zugleich die These, dass perfektive Verben des Polnischen eine gequantelte Referenz (telisierende Wirkung) auf inkrementelle Nomina aufweisen. Bei den deutschen neutralen (atelischen) Verben ist die Richtung umgekehrt: Das durch Determinatoren bestimmte Objekt bewirkt die Quantelung (Telizität) des Verbs.

\section{Abkürzungen}

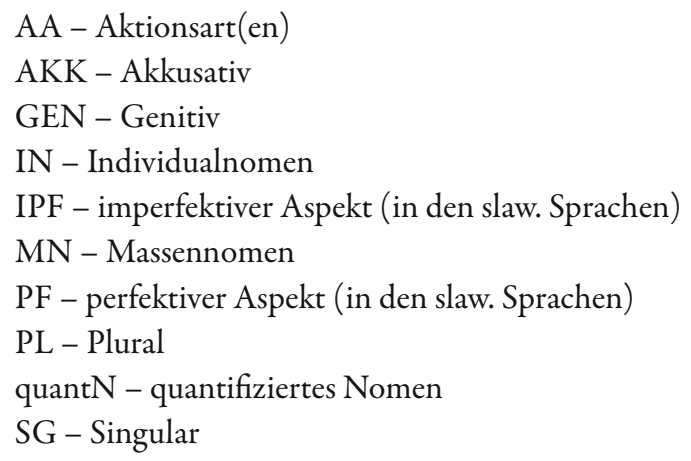

\section{Quellen mit Siglen}

Asche - Andrzejewski, J.: Popiót i diament. Warszawa 1968.

Asche und Diamant, übers. von H. Bereska. Leipzig 1974.

Iwasz - Iwaszkiewicz, J.: Zmowa mężczyzn. Warszawa 1975.

Verschwörung der Männer, übers. von C. Rymarowicz. Berlin 1982.

fremd - Hein, Ch.: Der fremde Freund. Berlin, Weimar 1986.

Obcy przyjaciel, übers. von B. Tarnas. Warszawa 1985.

Maria - Kuncewiczowa, M.: Zmowa nieobecnych. Warszawa 1978.

Die Verschwörung der Abwesenden, übers. von R. Matwin-Buschmann. Berlin, Weimar 1989.

Orph. - Schneidereit, O.: Der Orpheus von Paris. Berlin 1975.

Paryski Orfeusz, übers. von H. P. Anders. Poznań 1975.

Przedw. - Żeromski, S.: Przedwiośnie. Warszawa 1973.

Vorfrühling, übers. von K. Harrer und E. Thiele. Leipzig 1975.

Quo - Sienkiewicz, H.: Quo vadis? Warszawa 1983.

Quo vadis? Übers. von J. Bolinski. Berlin.

Quo vadis? Übers. von E. und R. Ettlinger. Einsiedeln (o. J.) 


\section{Bibliographie}

Abraham, Werner (1997): The interdependence of case, aspect, and referentiality in the history of German: the case of the verbal genitive. In: VAn Kemande, Ans / Vincent, Nigel (eds.): Parameters of morphosyntactic change. Cambridge: CUP, 29-61.

Agrell, Sigurd (1908): Aspektänderung und Aktionsartbildung beim polnischen Zeitworte. Lund: Håkan Ohlssons Buchdruckerei.

Andersson, Sven-Gunnar (1972): Aktionalität im Deutschen. Eine Untersuchung unter Vergleich mit dem russischen Aspektsystem. Uppsala: Almqvist \& Wiksell.

Avilova, Natalja S. (1976): Vid glagola i semantika glagolnogo slova. Moskva.

BERger, Tilman (2013): Imperfektive Verben in Handlungsfolgen im Westslavischen. In: KEMPGEN, Sebastian (Hg.): Deutsche Beiträge zum 15. Internationalen Slavistenkongress Minsk 2013. München: Peter Lang, 57-66.

Birkenmaier, Willy (1979): Artikelfunktionen in einer artikellosen Sprache. München: Wilhelm Fink.

Dickey, Stephen M. (2000): Parameters of Slavic Aspect. A Cognitive Approach. Stanford: CSLI Publications.

Duden (2016): Die Grammatik. Bd. 4; 9. überarbeitete Auflage. Berlin: Dudenverlag.

Ek, Britt-Marie (1996): Das deutsche Präsens. Tempus der Nichtvergangenheit. Stockholm: Almquist \& Wiksell.

EngeL, Ulrich u.a. (1999): Deutsch-polnische kontrastive Grammatik. Heidelberg: Groos.

FASSKe, Helmut (1981): Grammatik der obersorbischen Schriftsprache der Gegenwart. Morphologie. Bautzen: Domowina.

Filip, Hana (1999): Aspect, Eventuality Types and Nominal Reference. New York; London: Garland Publishing.

FleischHauer, Jens / CZARDybon, Adrian (2016): The Role of verbal prefixes and particles in aspectual composition. In: Studies in Language 40/1, 176-203.

Gladrow, Wolfgang (1979): Die Determination des Substantivs im Russischen und Deutschen. Leipzig: Enzyklopädie.

HeIndL, Olga (2017): Aspekt und Genitivobjekt. Tübingen: Stauffenburg.

Helbig, Gerhard / Buscha, Joachim (2001): Deutsche Grammatik. Berlin: Langenscheidt.

Is Ǎ̌EnKo, Alexander V. (1962): Die russische Sprache der Gegenwart. Teil I. Formenlehre. Halle (Saale): Niemeyer.

JakoBson, Roman ([1936] 1971): Beitrag zur allgemeinen Kasuslehre. In: Selected Writings vol. II. The Hague: Mouton.

Jespersen, Otto ([1924] 1965): The Philosophy of Grammar. New York: The Norton Library.

KĄTNy, Andrzej (1994): Zu ausgewählten Aktionsarten im Polnischen und deren Entsprechungen im Deutschen. Rzeszów: Wydawnictwo WSP.

KĄTNY, Andrzej (2000): Zu den Distributiva im Polnischen aus kontrastiver Sicht. In: KĄTNY, Andrzej (Hg.): Aspektualität in germanischen und slawischen Sprachen. Poznań: Wydawnictwo Naukowe UAM.

KĄTNy, Andrzej (2018): Aktionsart. In: Schierholz, Stefan (Hg.): Wörterbücher zur Sprachund Kommunikationswissenschaft (WSK) Online. De Gruyter, https://doi.org./10.1515/ wsk.1.1.aktionsart (17.07.2019). 
Koschmieder, Erwin ([1934] / 1987): Aspektologie des Polnischen (aus dem Polnischen übersetzt). Neuried: Hieronymus Verlag.

Kotin, Michail L. (2015): Plötzlich sehe ich einen Wolf! Über die Verwendung des historischen Präsens als eine Temporalitäts-Aspektualität-Schnittstelle. In: Kwartalnik Neofilologiczy H. 1, 3-16 (auch im Internet zugänglich).

KRIFKA, Manfred (1989): Nominalreferenz, Zeitkonstitution, Aspekt, Aktionsart. In: ABRAHAM, Werner / Janssen, Theo (Hg.): Tempus, Aspekt, Modus. Tübingen: Niemeyer, 227-258.

KRIFKA, Manfred (1989a): Nominalreferenz und Zeitkonstitution. Zur Semantik von Massentermen, Pluraltermen und Aspektklassen. München: W. Fink.

LeIss, Elisabeth (1992): Die Verbalkategorien des Deutschen. Berlin, New York: De Gruyter.

LeIss, Elisabeth (2000): Artikel und Aspekt. Berlin; New York: De Gruyter.

LöвEL, Elisabeth (1986): Apposition und Komposition in der Quantifizierung. Tübingen: Niemeyer.

Lyons, John ([1977] 1983): Semantik. vol. 2. München: Beck.

Quine, Willard (1960): Word and Object. Cambridge, Mass.: MIT Press.

SADZIŃSKI, Roman (1995/96): Die Kategorie der Determiniertheit und Indeterminiertheit im Deutschen und im Polnischen. Częstochowa: Wydawnictwo WSP.

SCHWENK, Hans-Jörg (2007): Telisch und atelisch: aspektual oder aktional oder beides? Zeno Vendlers Verbklassifikation und ihr Verhalten zu den Kategorien Aspekt und Aktionsart. Arbeitspapier Nr. 53 (Neue Folge). Köln.

Steinitz, Renate (1981): Der Status der Kategorie „Aktionsart" in der Grammatik (oder: Gibt es Aktionsarten im Deutschen?). Berlin: Akademie der Wissenschaften.

Vater, Heinz ( $\left.{ }^{2} 1979\right)$ : Das System der Artikelformen im gegenwärtigen Deutsch. Tübingen: Niemeyer.

Vendler, Zeno (1957): Verbs and times. In: The Psychological Rewiev 66/2, 143-160.

VerkuYL, Henk (1972): On the Compositional Nature of the Aspects. Dordrecht: Reidel.

VERKUYL, Henk (1993): A Theory of Aspectuality. The Interaction between temporal and atemporal Structure. Cambridge: CUP.

Weinrich, Harald (1993): Textgrammatik der deutschen Sprache. Mannheim: Dudenverlag.

WierzBicka, Anna (1967): On the semantics of verbal aspect in Polish. In: To Honor Roman Jakobson vol. 3. The Hague, Paris: Mouton, 2231-2249.

Wierzbicka, Anna (1988): The Semantics of Grammar. Amsterdam; Philadelphia: John Benjamins.

Zifonun, Gisela et al. (Hg.) (1997): Grammatik der deutschen Sprache. Bd. 3. Berlin: De Gruyter. 